\title{
Forecasting cosmic doomsday from CMB-LSS cross-correlations
}

\author{
Jaume Garriga \\ Departament de Fisica Fonamental, Universitat de Barcelona, Marti i Franques, 1, 08028 Barcelona, Spain \\ and Institute of Cosmology, Department of Physics and Astronomy, Tufts University, Medford, Massachusetts 02155, USA \\ Levon Pogosian \\ Institute of Cosmology, Department of Physics and Astronomy, Tufts University, Medford, Massachusetts 02155, USA \\ Tanmay Vachaspati \\ CERCA, Department of Physics, Case Western Reserve University, 10900 Euclid Avenue, Cleveland, Ohio 44106-7079, USA
}

(Received 30 November 2003; published 17 March 2004)

\begin{abstract}
A broad class of dark energy models, which have been proposed in attempts at solving the cosmological constant problems, predict a late time variation of the equation of state with redshift. The variation occurs as a scalar field picks up speed on its way to negative values of the potential. The negative potential energy eventually turns the expansion into contraction and the local universe undergoes a big crunch. In this paper we show that cross-correlations of the cosmic microwave background anisotropy and matter distribution, in combination with other cosmological data, can be used to forecast the imminence of such cosmic doomsday.
\end{abstract}

DOI: $10.1103 /$ PhysRevD.69.063511

PACS number(s): $98.80 . \mathrm{Cq}$

\section{INTRODUCTION}

Observational cosmology has yielded several surprises, of which the most perplexing is the discovery of a smooth dark energy (DE) component which has come to dominate the Universe at recent epochs, causing cosmic acceleration [1,2]. The nature of this component is still a matter of speculation, and a very important challenge for the coming years will be to determine its origin and physical properties. Recently, several groups [3-7] have reported positive results for the crosscorrelation between the cosmic microwave background (CMB) power spectrum and that of different large scale structure (LSS) surveys, providing further evidence for the existence of DE. In this paper, we shall try and illustrate how such cross-correlation may help in unveiling some of the properties of DE, focusing on the observational signatures of a model with a time dependent DE equation of state.

The simplest interpretation of the dark energy is in terms of a cosmological constant, with an equation of state $p_{D}$ $=-\rho_{D}$. The cosmological constant, however, raises two puzzles of its own. First, there is a glaring discrepancy between the observed dark energy density $\rho_{D}$ and the huge values of the cosmological constant suggested by particle physics models. Second, the observed $\rho_{D}$ is comparable to the matter density $\rho_{m}$. This is the notorious time coincidence problem: why do we happen to live at the epoch when the dark energy starts dominating?

It has long been suggested that both puzzles may find a natural explanation through anthropic selection effects, in scenarios where $\rho_{D}$ is a random variable, taking different values in different parts of the Universe. The proposed selection mechanism is very simple [8-14]. The growth of density fluctuations leading to galaxy formation effectively stops when $\rho_{D}$ comes to dominate over the matter density. In regions where $\rho_{D}$ is greater, it will dominate earlier, and thus there will be fewer galaxies (and therefore fewer observers). A typical observer should then expect to find herself in a region where $\rho_{D}$ dominates at about the epoch of galaxy formation (which is close to the present time). Much larger values of $\rho_{D}$ would yield no galaxies at all, while much smaller values are unlikely due to the smallness of the corresponding range of $\rho_{D}$, assuming that all values of $\rho_{D}$ are a priori equally likely $[11,15]$.

A simple implementation of this idea is obtained by assuming that the dark energy is due to a scalar field $\phi$ with a very flat potential $V(\phi)[15,16]$. The values of $\phi$ are randomized by quantum fluctuations during inflation, resulting in a variation of $\phi$ with a characteristic scale much greater than the present Hubble radius. Galaxy formation is possible only in regions where $V(\phi)$ is in a narrow range near $V$ $=0$. One expects that the potential in this range is well approximated by a linear function $[15,17,18]$,

$$
V(\phi)=-s \phi
$$

where $s \equiv-V^{\prime}(0)$ and we have set $\phi=0$ at $V=0$. The slope $s$ should be sufficiently small, so that the variation of $\phi$ is not fast on the present Hubble scale. Quantitatively, this can be expressed as the slow roll condition,

$$
s \lesssim 3 H_{0}^{2} M_{p},
$$

where $M_{p}=1 / \sqrt{8 \pi G}$ is the reduced Planck mass, $G$ is Newton's gravitational constant, and $H_{0}$ is the present Hubble expansion rate.

In models with a single DE field, and in the absence of ad hoc adjustments, it has been argued in [17] that the slow roll condition (2) is likely to be satisfied by excess, by many orders of magnitude, rather than marginally. In this case, $\phi$ remains nearly constant on the Hubble scale, and the effective equation of state for the dark energy is $w \equiv p_{D} / \rho_{D}$ $\approx-1$, with a very high accuracy. However, a different situation may be expected in multifield models, where the slope of the potential $s$ is itself a random variable $[19,21]$ [the role 
of the field $\phi$ in multifield models is played by the variable in the direction of $\nabla V\left(\phi_{a}\right)$ in the field space]. The observed value of the slope may then be influenced by anthropic selection. A very large slope would cause a big crunch much before any observers can develop. Hence, in cases where the prior distribution favors large $s$, the most probable values of the slope would be the ones for which the slow roll condition (2) is only marginally satisfied [21] (see also [18]).

We thus have some motivation to consider a model where the dark energy is due to a scalar field with a linear potential (1) and a slope $s$ marginally satisfying the slow roll condition (2). A marginal value of the slope implies that the big crunch will be imminent about 10 billion years from now. The model can therefore be called a "doomsday model." A salient feature of this scenario is that the equation of state of dark energy changes significantly at low redshift, when the correlation between the large scale structure evolution and the CMB temperature anisotropies develops. Hence, we may expect that the analysis of such cross-correlation may reveal a time varying equation of state $p_{D}=w(z) \rho_{D}$, where $z$ is the redshift. This will be the subject of the present paper.

Prospective constraints on cosmic doomsday based on future determinations of the dimming of distant supernovae were discussed in Ref. [22]. The analysis shows that SNIa observations, in combination with $\mathrm{CMB}$ and weak lensing data, have an impressive potential for constraining the equation of state parameter. However, the constraints reported in [22] still show a considerable degeneracy amongst models with the same "average" $\langle w\rangle$ [see Eq. (8) below]. Interestingly, as we shall see, the ISW-matter cross-correlation breaks this degeneracy, offering the possibility of telling a true doomsday model from a model with a constant $w=\langle w\rangle$.

The methods presented here can obviously be used in a more general context, provided that there is significant evolution of $w$ at low redshifts. A rather common assumption in phenomenological studies of dark energy is to consider the simplest case of a constant $w$. This is partially motivated by degeneracies such as the one we just discussed above, which also occur in the angular spectrum of CMB anisotropies [23] as well as in the linear matter power spectrum. The analysis of CMB-LSS cross correlations with a constant $w$ (including $w=-1)$ was considered in Refs. [24-27]. Here we shall drop this assumption, since the variation of $w$ with redshift may provide a very exciting clue to the nature of dark energy, as discussed above.

In our calculation we shall adopt a top-down approach, starting from the primordial spectrum of fluctuations. This differs from previous studies where the starting point is the present matter power spectrum (which is evolved backward in order to find its correlation with $\mathrm{CMB}$ ). Our approach unifies the treatment of $\mathrm{CMB}$ and matter power spectra, and is more convenient for taking full account of fluctuations in the dark energy. Moreover, since all perturbations are evolved numerically with the CMBFAST code [28], we do not resort to the frequently used approximate analytical expressions for the growth function, or the also commonly used small angle approximation [29]. The details of our calculation are reported in the Appendix.
The paper is organized as follows. In Sec. II we describe the evolution of the Universe according to the doomsday model. We also show that the corresponding CMB and present matter power spectra are virtually identical to those which are obtained in a model with a constant $w$, equal to the average $\langle w\rangle$ for the doomsday model. In Sec. III we study the matter/CMB temperature cross-correlation, and show that it can be used to break this degeneracy. Our conclusions are summarized in Sec. IV.

\section{EVOLUTION AND POWER SPECTRA IN THE DOOMSDAY SCENARIO}

We are interested in the late time evolution of our observable Universe, and so we assume a background model which is homogeneous and isotropic. In addition to the scalar field $\phi$ with linear potential (1), the Universe contains the usual radiation and matter. The dynamics is given by the Friedman-Robertson-Walker (FRW) equation and the scalar field equation

$$
\begin{gathered}
H^{2} \equiv\left(\frac{\dot{a}}{a}\right)^{2}=\frac{1}{3 M_{p}^{2}}\left(\frac{\dot{\phi}^{2}}{2}-s \phi\right)+\frac{\Omega_{m 0} H_{0}^{2}}{a^{3}}, \\
\dot{\phi}+3 H \dot{\phi}-s=0 .
\end{gathered}
$$

Here $\Omega_{m 0}$ is the fractional energy density in matter today, and $H_{0}$ is the present Hubble rate.

In addition to the equations of motion, we need to specify initial or boundary conditions. At early times $(t \rightarrow 0)$, we expect that the scalar field is at rest and so $\dot{\phi}=0$. This is because, in the back of our minds, we imagine an inflationary phase which redshifts the gradients and velocities of the scalar field. Hence the scalar field is effectively homogeneous and static at $t=0$. The initial value of the scale factor is zero as in usual Friedmann-Robertson-Walker evolution. The initial value of the scalar field is a free parameter. As mentioned in the Introduction, this takes different values in distant regions of the Universe, separated by distances much larger than the present Hubble radius. Finally, we require that the present value of the total energy density be unity. This is a boundary condition. These conditions can be summarized as follows:

$$
a(0)=0, \quad \phi(0)=\phi_{0}, \dot{\phi}(0)=0, H^{2}\left(t_{0}\right)=H_{0}^{2},
$$

where $t_{0}$ is the present time, defined by the requirement that $a\left(t_{0}\right)=1$.

The cosmological evolution following from Eqs. (3), (4) and (5) has been studied by several authors [17,20-22]. The main features of the evolution are as follows:

(i) The Universe starts out dominated by matter and hence $a \sim t^{2 / 3}$. At the same time the scalar field is essentially at rest.

(ii) After some time, the matter density falls below that of the scalar field potential energy, and the evolution becomes scalar field dominated. Since most of the energy in the scalar field is potential energy, we have $a \propto \exp (H t)$.

(iii) As the field slips down the potential, the potential energy changes sign once $\phi$ changes sign. With further slip- 


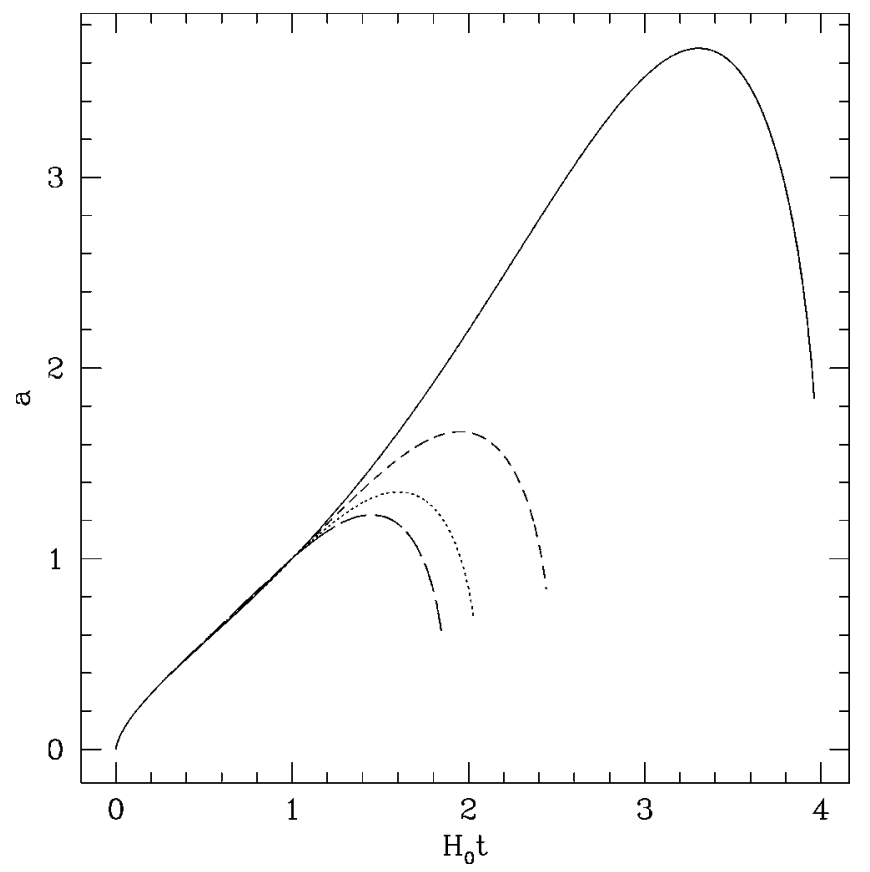

FIG. 1. Scale factor versus time for four different slopes: $\tilde{s}=1$ (solid), $\tilde{s}=2$ (short dash), $\tilde{s}=3$ (dot) and $\tilde{s}=4$ (long dash).

ping, a time comes when the total energy density is zero. This epoch marks the turning point, where cosmic expansion changes to contraction. From this time on, $H$ is given by the negative square root of the right-hand side of Eq. (3).

(iv) As the Universe starts contracting, the kinetic energy of the scalar field comes to dominate. But there is no stopping the contracting phase, and the Universe rapidly arrives at the big crunch.

These features are apparent in Fig. 1, where the time evolution of the Universe is represented for different values of the dimensionless slope

$$
\tilde{s} \equiv s /\left(\sqrt{3} M_{p} H_{0}^{2}\right) .
$$

One feature of this evolution that is relevant for observational cosmology is that the equation of state for the scalar field changes in an unconventional manner. Very early on, $\dot{\phi} \rightarrow 0$ and hence $p_{D} \sim-\rho_{D}$ where $p_{D}$ and $\rho_{D}$ denote pressure and energy density in $\phi$. The dark energy equation of state parameter is defined by

$$
w \equiv \frac{p_{D}}{\rho_{D}}=\frac{\dot{\phi}^{2} / 2-s \phi}{\dot{\phi}^{2} / 2+s \phi} .
$$

Since $\dot{\phi} \simeq 0$ at early times, $w \simeq-1$. At later times, the field starts to roll down the potential and hence the kinetic energy starts to play a role in $p_{D}$ and $\rho_{D}$. This means that $w$ increases with time. In Fig. 2 we show $w$ as a function of redshift $z$ (figures analogous to Figs. 1 and 2 can also be found in $[18,22])$.

If we only varied $\tilde{s}$, keeping all other cosmological parameters fixed, we would change the angular diameter distance to the last scattering surface and spoil the agreement

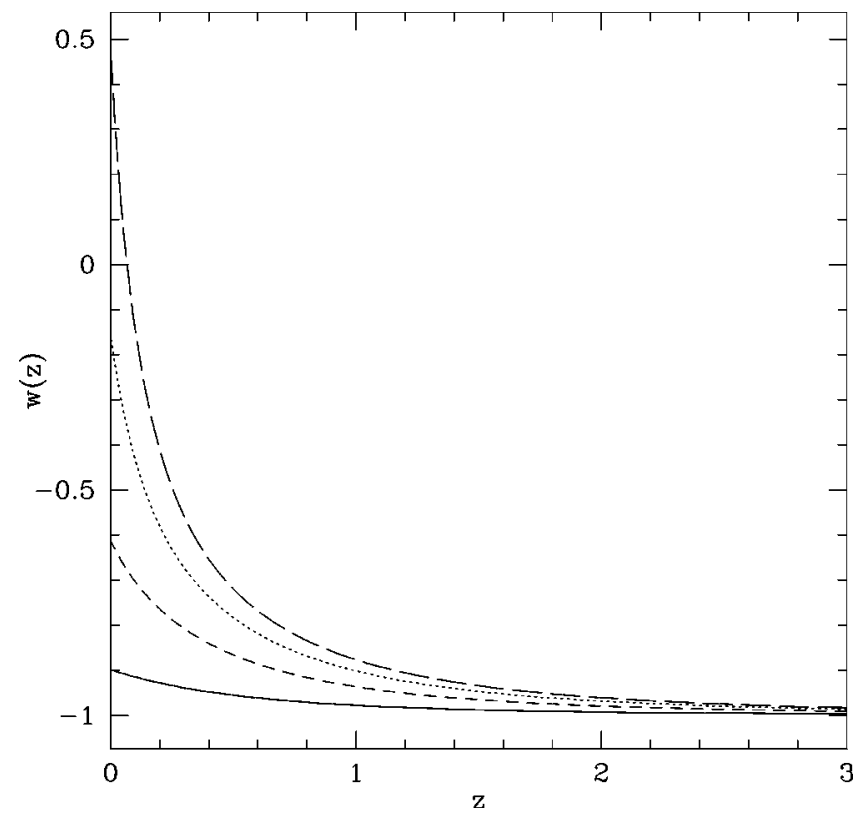

FIG. 2. $w(z)$ versus $z$ for the four different slopes considered in Fig. 1.

with current $\mathrm{CMB}$ data. Hence it is necessary to suitably adjust cosmological parameters for different values of $\tilde{s}$. The cosmological parameters in models with different values of $\tilde{s}$, presented in Figs. 1 and 2, depend on $\tilde{s}$. The simplest way to preserve the shape of the CMB fluctuations spectra on small and intermediate scales (where the cosmic variance is small) is to explore the so-called geometric degeneracy [30]. The shape of the spectra depends mainly on two scales: $r_{s}$ - the sound horizon at the time of recombination, and $d_{A}$ - the angular diameter distance to the last scattering surface. Models with the same values of $\Omega_{M} h^{2}$ and $\Omega_{b} h^{2}$ have the same $r_{s}$. Here $\Omega_{M}$ and $\Omega_{b}$ are the total matter and baryon density fractions today, and $h=H_{0} /\left(100 \mathrm{~km} \mathrm{sec}^{-1}\right.$ $\mathrm{Mpc}^{-1}$ ). Since the dark energy did not play a significant role at the time of recombination, changing the value of $\tilde{s}$, or $w$, does not affect $r_{s}$. The main effect that changes in the properties of the dark energy have on the CMB spectra on small angular scales is due to the change in $d_{A}$, which manifests itself as a shift in the positions of the peaks in the angular spectra. This shift can be compensated for, without altering the structure of the peaks, by adjusting the value of $h$. As a reference model we use the Wilkinson Microwave Anisotropy Probe's (WMAP's) best fit power law cold dark matter with a cosmological constant ( $\Lambda$ CDM) model [31] with $h$ $=0.72$, spectral index $n=0.99$, reionization optical depth $\tau_{r}=0.166, \Omega_{b} h^{2}=0.024, \Omega_{M} h^{2}=0.14$ and amplitude $A$ $=0.86$ (as defined in [32]). Given a value of $\tilde{s}$, we vary the value of $h$, while keeping $\tau_{r}, A, n, \Omega_{M} h^{2}$ and $\Omega_{b} h^{2}$ fixed, and find one that best reproduces the CMB spectra of the reference model.

As was noted in [23], except for the very large scales, CMB spectra are only sensitive to the averaged value of the equation of state of dark energy, $\langle w\rangle$, defined as 
TABLE I. Models considered in the paper. $\tilde{s}$ is the dimensionless slope of the potential, defined in Eq. (6). For each value of $\tilde{s}$, the dimensionless Hubble parameter $h$ is adjusted so that the model reproduces the CMB peak structure observed by WMAP. The table also shows the average value of $w$ for different models.

\begin{tabular}{lcc}
\hline \hline Model & $\mathrm{h}$ & $\langle w\rangle_{\left[0, z_{l s}\right]}$ \\
\hline$\tilde{s}=0$ & 0.72 & -1 \\
$\tilde{s}=1$ & 0.69 & -0.94 \\
$\tilde{s}=2$ & 0.66 & -0.81 \\
$\tilde{s}=3$ & 0.62 & -0.66 \\
\hline \hline
\end{tabular}

$$
\langle w\rangle_{\left[0, z_{l s}\right]}=\frac{\int_{a_{l s}}^{1} d a \Omega_{D}(a) w(a)}{\int_{a_{l s}}^{1} d a \Omega_{D}(a)},
$$

where the subindex $l s$ on $z$ and $a$ refers to the surface of last scattering. In Table I we show the best fit values of $h$ and the corresponding values of $\langle w\rangle_{\left[0, z_{l s}\right]}$ for several values of $\tilde{s}$. Note that the uncertainty in WMAP's estimate of $h$ was about \pm 0.05 [31]. The same uncertainty would apply to the best fit values of $h$ corresponding to models with $\tilde{s} \neq 0$.

In the doomsday model, the equation of state parameter can vary significantly at recent redshifts. This variation is not necessarily constrained by existing analysis of supernova data [33,34], which by and large assumed a constant $w$. In Fig. 3 we plot the effective luminosity $m^{\text {eff }}$ as a function of redshift, as defined in [2], for the $\tilde{s}=2$ and $w=-0.81$, and $\tilde{s}=3$ and $w=-0.66$ models. As one can see from the figure, the doomsday model with a given $\tilde{s}$ and the corresponding constant $w$ model have almost identical predictions in the magnitude versus redshift curves. Since constant $w$ models with $w>-0.8$ are disfavored at the $1 \sigma$ level [33], the value $\tilde{s}=3$ which gives an average $w$ of -0.66 is also disfavored at the $1 \sigma$ level. However, we shall still include it in our subsequent analysis, since it is not excluded at the $2 \sigma$ level [33].

In Fig. 4 we plot the temperature (TT) and temperaturepolarization cross-correlation (TE) spectra for models in Table I. As shown in Table I, as we increase $\tilde{s}$, a smaller value of $H_{0}$ is needed in order to fit the CMB spectra. ${ }^{1}$ The value $h=0.62 \pm 0.05$ which we used for the $\tilde{s}=3$ model is somewhat lower than the currently favored observational $1 \sigma$ region, given by $h=0.72 \pm .08$ [35], but still marginally consistent with it.

\footnotetext{
${ }^{1}$ In principle, one could vary other cosmological parameters as well. However, unless one changed the model considerably, e.g. relaxed the assumptions of adiabaticity or scale-invariance of primordial fluctuations, it is unlikely that one could avoid making $H_{0}$ small in models with $\langle w\rangle>-1$ [31].
}

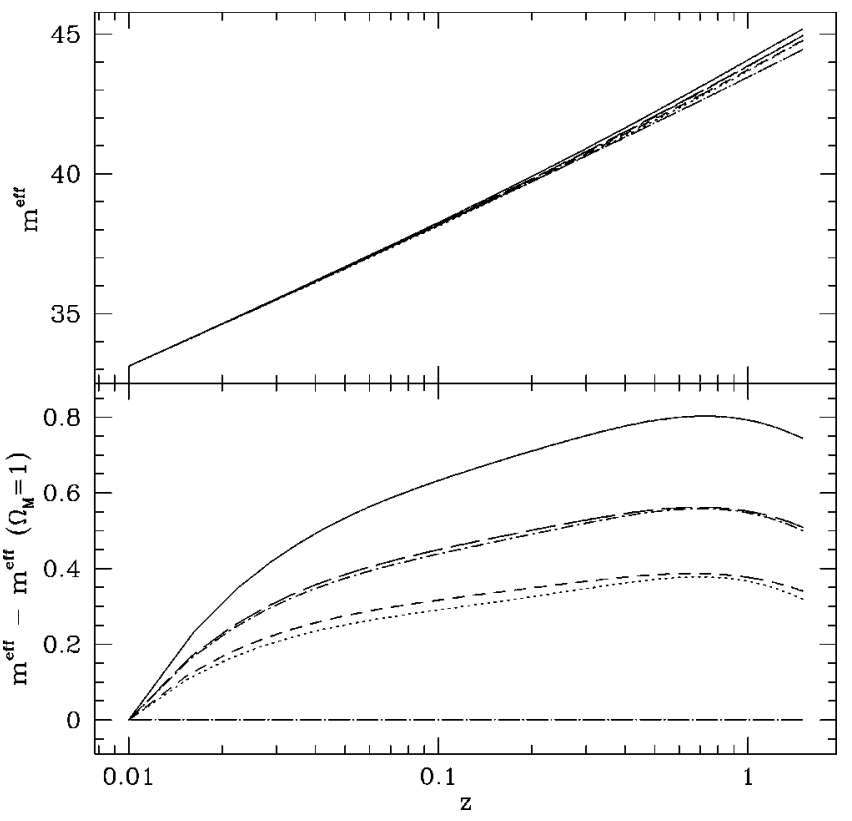

FIG. 3. Upper panel: the luminosity, $m^{\text {eff }}$, vs redshift plots for the $\Lambda \mathrm{CDM}$ (solid line), $\tilde{s}=2$ (dot-short dash), $\tilde{s}=3$ (dot), $w=$ -0.81 (long dash), $w=-0.66$ (short dash) and the $\Omega_{M}=1$ (dot long dash) models. Lower panel: differences between the $m^{\text {eff }}$ for the models in the upper panel and the $m^{e f f}$ for the $\Omega_{M}=1$ model. Models with the same average value of $w$ are practically indistinguishable.

The equation of state parameter $w(z)$ obviously has an impact on different observables, such as CMB and matter power spectra. In particular, it should affect the integrated Sachs-Wolfe (ISW) contributions to CMB anisotropies. At first sight, one may think that a less negative equation of state for dark energy would result in a suppression of ISW, relative to the case of a cosmological constant. In a universe dominated by matter, with pressure $p_{M}=0$, there is no time dependence of the gravitational potential, and the frequency of a photon is only redshifted by the cosmological expansion. In this case, there is no late ISW effect. In the doomsday scenario, the dark energy equation of state is closer to that of ordinary matter, and one might expect that the ISW effect would be smaller than in the $w=-1$ case. However, the dark energy perturbations are coupled to the dark matter perturbations, and will also contribute to the ISW effect. The net result is that there is no suppression of the ISW effect even for values of $\tilde{s}$ corresponding to average $w$ as high as -0.66 . This is illustrated in Figs. 5 and 6. There, we plot the angular spectrum $C^{T T, I S W}$ and the autocorrelation function $C^{T T, I S W}(\theta)$ of temperature anisotropy due to the late ISW effect, defined as

$$
\begin{aligned}
C^{T T, I S W}(\theta) & \equiv\left\langle\Delta T_{I S W}\left(\hat{n}_{1}\right) \Delta T_{I S W}\left(\hat{n}_{2}\right)\right\rangle \\
& =\sum_{l=0}^{\infty} \frac{2 \ell+1}{4 \pi} C^{T T, I S W}{ }_{\ell} P_{\ell}(\cos \theta) .
\end{aligned}
$$

Here $\Delta T_{I S W}(\hat{n})$ is the ISW contribution to the temperature anisotropy in the direction $\hat{n}$ on the sky, $\theta$ is the angle be- 


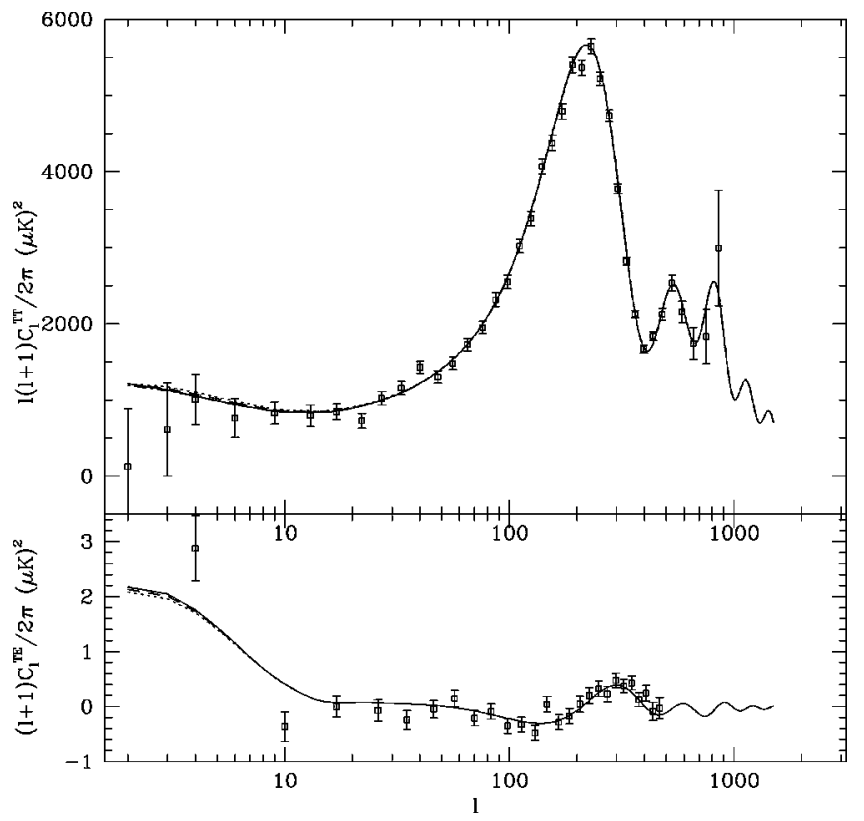

FIG. 4. TT and TE angular spectra for $\tilde{s}=0$ ( $\Lambda \mathrm{CDM})$ (solid line), $\tilde{s}=1$ (dot-short dash), $\tilde{s}=2$ (short dash) and $\tilde{s}=3$ (dot) models together with the WMAP data points. The value of $h$ is chosen as indicated in Table I. Note that due to the geometric degeneracy, all curves look very much alike, the effect of a higher $\tilde{s}$ being undone by choosing a lower $h$.

tween directions $\hat{n}_{1}$ and $\hat{n}_{2}$, and the angular brackets denote ensemble averaging [the expression for $\Delta T_{I S W}(\hat{n})$ is given in the Appendix]. Note that the late ISW contribution of most models is in fact a bit larger than in the $\Lambda$ CDM case.

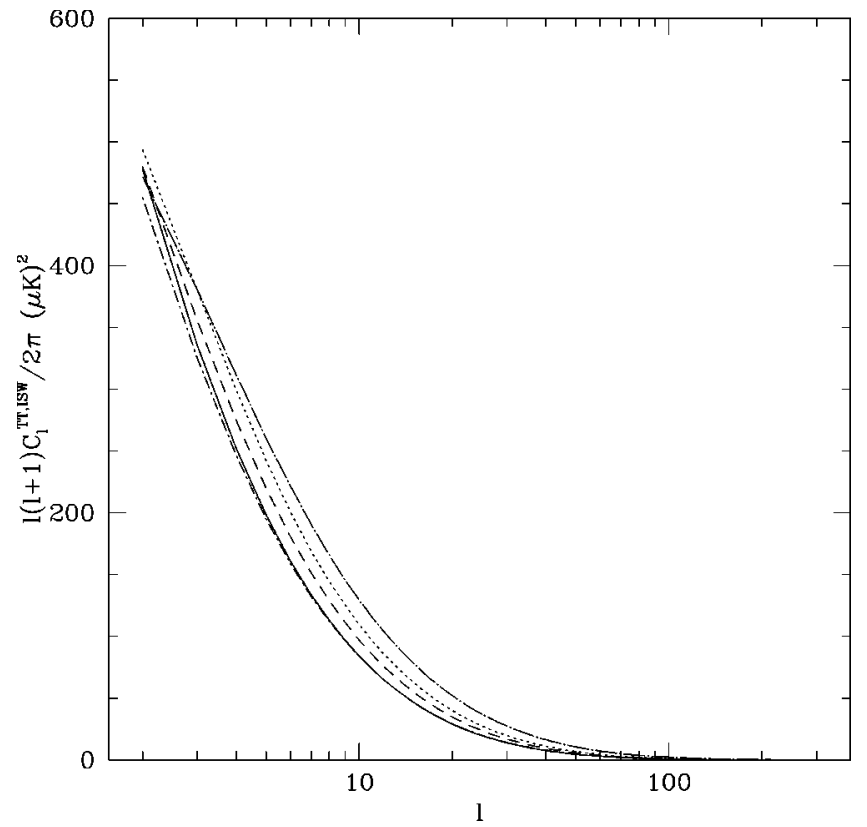

FIG. 5. The CMB temperature anisotropy angular spectrum due to the late ISW effect, $C^{T T, I S W}{ }_{\ell}$, for the five models in Fig. 4 as well as for the model with the constant $w=-0.66$ (dot-long dash line). As explained in the text, there is no suppression of the ISW effect due to a higher value of $w$.

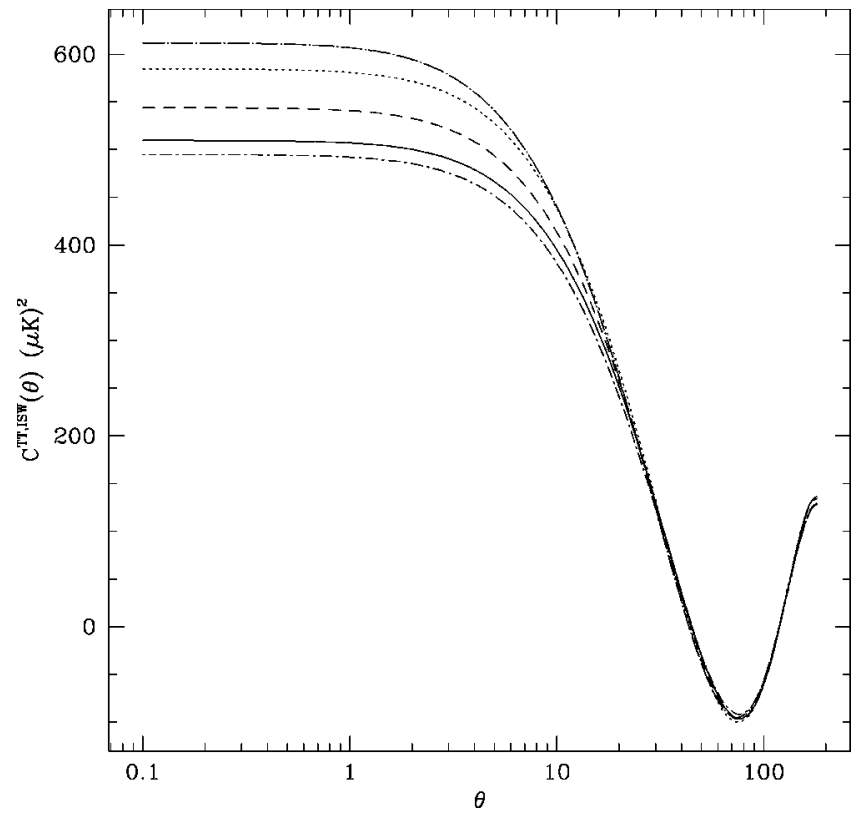

FIG. 6. The ISW sourced temperature anisotropy correlation function $C^{T T, I S W}(\theta)$ for the models in Fig. 5.

It is quite clear from Fig. 4 that CMB spectra alone are not capable of differentiating between models with different values of $\tilde{s}$ (including $\tilde{s}=0$ ) because of the geometric degeneracy. The effect of a larger $\tilde{s}$ can be undone with a smaller $h$. (Of course, if we had a stronger observational constraint on $h$, then this would result in stronger constraints on $\tilde{s}$.) CMB spectra alone also cannot differentiate between a model with a certain value of $\tilde{s}$ and a model with the corresponding constant $w=\langle w\rangle_{\left[0, z_{l s}\right]}$ (see Table I). This is illustrated in Fig. 7, where we plot the predictions of the $\Lambda \mathrm{CDM}$,

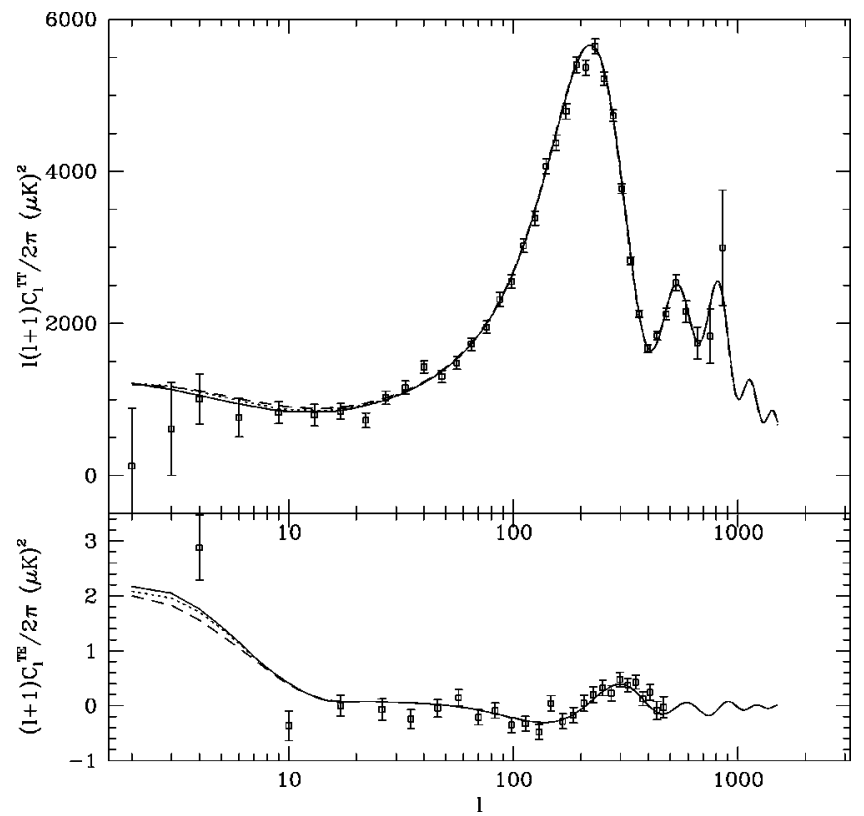

FIG. 7. $C_{l}$ vs $l$ for $\Lambda \mathrm{CDM}$ (solid), $s=3$ (dot) and $w=-0.66$ (dash) models. 


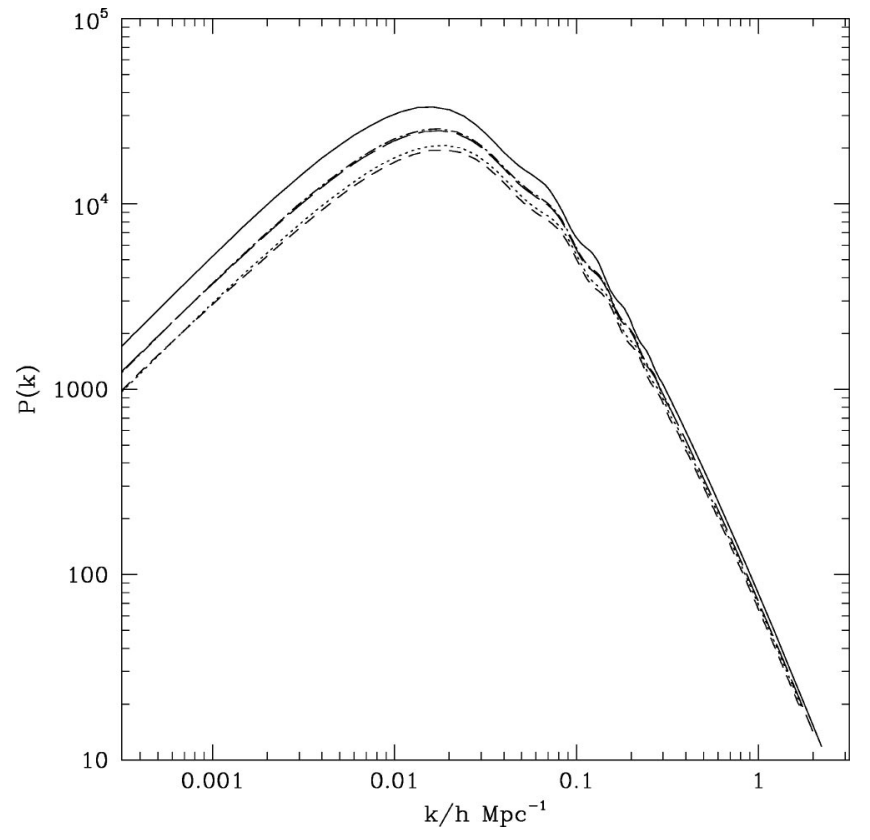

FIG. 8. The present linear power spectrum $P(k)$ vs $k$ is sensitive to $\langle w\rangle$, but a model with a constant $w$ has virtually the same $P(k)$ as a doomsday model with the same $\langle w\rangle$. Here, we plot $P(k)$ for the models in Fig. 7, as well as for the $\tilde{s}=2$ (dot-short dash) and the $w=-0.81$ (long dash) models.

$w=$ const $=-0.66$ and $\tilde{s}=3$ models. Ideally, some of the degeneracy can be removed by considering the matter power spectrum. Assuming that we had a good control over the bias, the matter power spectrum could in principle be inferred from observations. In Fig. 8 we plot the linear matter power spectra at $z=0$ for the $w=$ const $=-0.66$ and $\tilde{s}=3$ models, as well as for the $\tilde{s}=2$ and its corresponding $w$ $=$ const $=-0.81$ model. Note that $P(k)$ differs substantially on large scales for $\Lambda \mathrm{CDM}$ and the models with a lower average value of $w$. However, there is still an impressive degeneracy between models with the same $\langle w\rangle$ : the curve corresponding to a constant $w=-0.66$ and the curve corresponding to the model with $\tilde{s}=3$ (with $\langle w\rangle=-0.66$ ) are almost identical, and similarly for the $\tilde{s}=2$ and the $w$ $=-0.81$ models. In the next section we shall concern ourselves with breaking this residual degeneracy.

\section{ISW AND TEMPERATURE-MATTER DENSITY CORRELATIONS}

In this section we show how the CMB-LSS crosscorrelation can be used to probe the time-dependence of the dark energy equation of state. The cross-correlation is defined as

$$
C^{M T}(\theta) \equiv\left\langle\Delta\left(\hat{\mathbf{n}}_{1}\right) \delta\left(\hat{\mathbf{n}}_{2}\right)\right\rangle=\sum_{l=2}^{\infty} \frac{2 \ell+1}{4 \pi} C^{M T}{ }_{\ell} P_{\ell}(\theta),
$$

where $\Delta\left(\hat{\mathbf{n}}_{1}\right)$ and $\delta\left(\hat{\mathbf{n}}_{2}\right)$ are the CMB temperature anisotropy and the matter density contrast along directions $\hat{\mathbf{n}}_{1}$ and $\hat{\mathbf{n}}_{2}$ separated by the angle $\theta$ on the sky. ${ }^{2}$ In the Appendix we show that the angular cross-correlation spectrum $C^{M T}{ }_{\ell}$ can be written as

$$
C_{\ell}^{M T}=4 \pi \frac{9}{25} \int \frac{d k}{k} \Delta_{\mathcal{R}}^{2} T^{I S W}{ }_{\ell}(k) M_{\ell}(k),
$$

were $\Delta_{\mathcal{R}}^{2}$ is the primordial curvature power spectrum, as defined, e.g. in [36], and $T^{I S W}(k)$ and $M_{\ell}(k)$ are given by

$$
\begin{gathered}
T^{I S W}{ }_{\ell}=\int_{\eta_{r}}^{\eta_{0}} d \eta e^{-\tau(\eta)} j_{\ell}\left(k\left[\eta-\eta_{0}\right]\right)\left(c_{\Phi \Psi} \dot{\phi}-\dot{\psi}\right), \\
M_{\ell}=c_{\delta \Psi} \int_{\eta_{r}}^{\eta_{0}} d \eta j_{\ell}\left(k\left[\eta-\eta_{0}\right]\right) \dot{z} W_{g}[z(\eta)] \widetilde{\delta}(k, \eta),
\end{gathered}
$$

where the dot denotes differentiation with respect to conformal time $\eta, \eta_{0}$ is the time today, $\eta_{r}$ is a time very early in the radiation era, $\tau(\eta)$ is the opaqueness, $W_{g}(z)$ is the normalized galaxy selection function, $\dot{\phi}(k, \eta), \dot{\psi}(k, \eta)$ and $\widetilde{\delta}(k, \eta)$ are evolution functions which we define in the Appendix and which can be calculated numerically using CMBFAST [28], $c_{\delta \Psi}$ and $c_{\Phi \Psi}$ are numerical coefficients also defined in the Appendix, and $j_{\ell}(\cdot)$ are spherical Bessel functions.

The choice of the selection function $W_{g}(z)$ depends on which large scale structure data set one wants to consider. Depending on the particular experiment, one also has to account for the possible bias between the distribution of the observed objects and that of the underlying dark matter. Our results for $C^{M T}(\theta)$ for the $\Lambda \mathrm{CDM}$ model are consistent with those of [4-6], when appropriate biases and selection functions are used.

Equation (11) differs from the analogous expressions in [4-6,24-27] as it uses the primordial curvature power spectrum rather than today's matter power spectrum. This, as explained in the Appendix, allows us to take a more complete account of the dark energy perturbations.

$C^{M T}(\theta)$ can be particularly useful for differentiating between $w=$ const and varying $w$ models. The cross-correlation is sensitive to the value of $w$ averaged over the range of the window function $W_{g}(z)$. If $w$ is a rapidly changing function of redshift, as in the case of the doomsday model, then depending on where the maximum of the selection function is, $C^{M T}(\theta)$ will "see" different values of $\langle w\rangle$. We have calculated the cross-correlation for several window functions, all taken to be Gaussians of approximately the same width as the SDSS window functions [6], all with the same standard deviation $\sigma_{w}=0.07$ and centered at various values of $z_{w}$ in the interval $[0.1,0.8]$. In Fig. 9 we show the plot of $C^{M T}(\theta)$

\footnotetext{
${ }^{2}$ The monopole and the dipole contributions depend on the choice of the reference frame and are not included.
} 


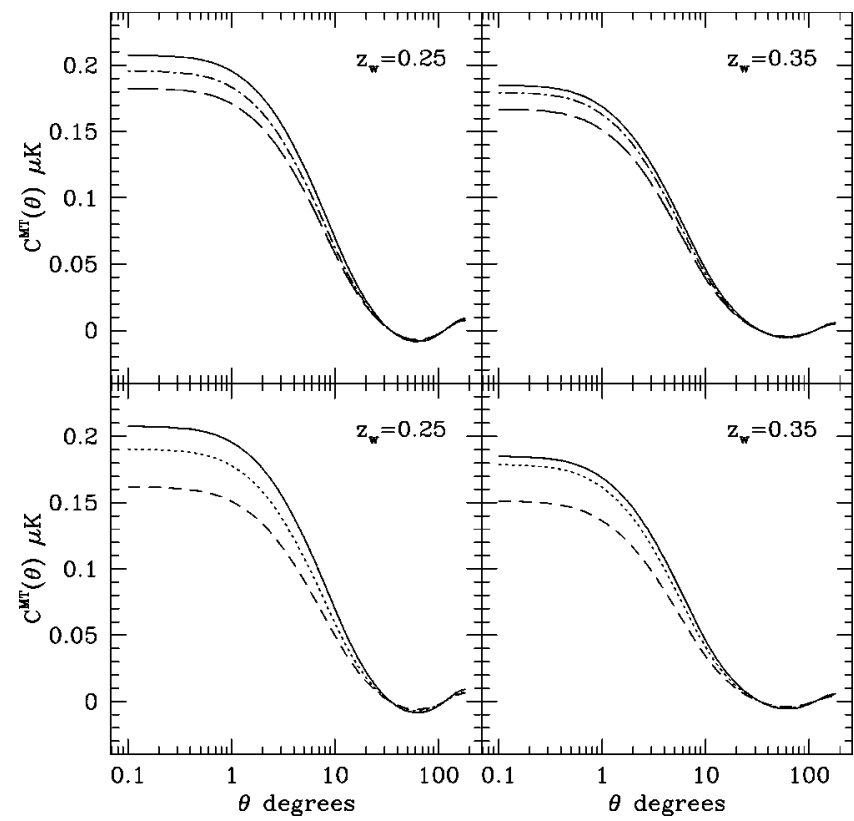

FIG. 9. The cross-correlation breaks the remaining degeneracies. In each panel, we plot a doomsday model together with its corresponding constant $w$ model [these two have degenerate CMB spectra and $P(k)]$. For comparison, each plot also contains the fiducial $\Lambda$ CDM model. Top two panels: $C^{M T}(\theta)$ for the $\tilde{s}=2$ (dotshort dash) and the $w=-0.81$ (long dash) models, as well as for the $\Lambda \mathrm{CDM}$ model (solid), for two values of $z_{w}$. Bottom two plots: the same as in the top two plots but for the $\tilde{s}=3$ (dot) and the $w$ $=-0.66$ (short dash) models.

for the $\tilde{s}=2$ and $\tilde{s}=3$ models, together with their respective $w=$ const models, at two different values of $z_{w}$. Corresponding angular spectra $C^{M T}$ are shown in Fig. 10. In addition, in Fig. 11 we plot the values of $C^{M T}\left(0.1^{\circ}\right)$ as a function of

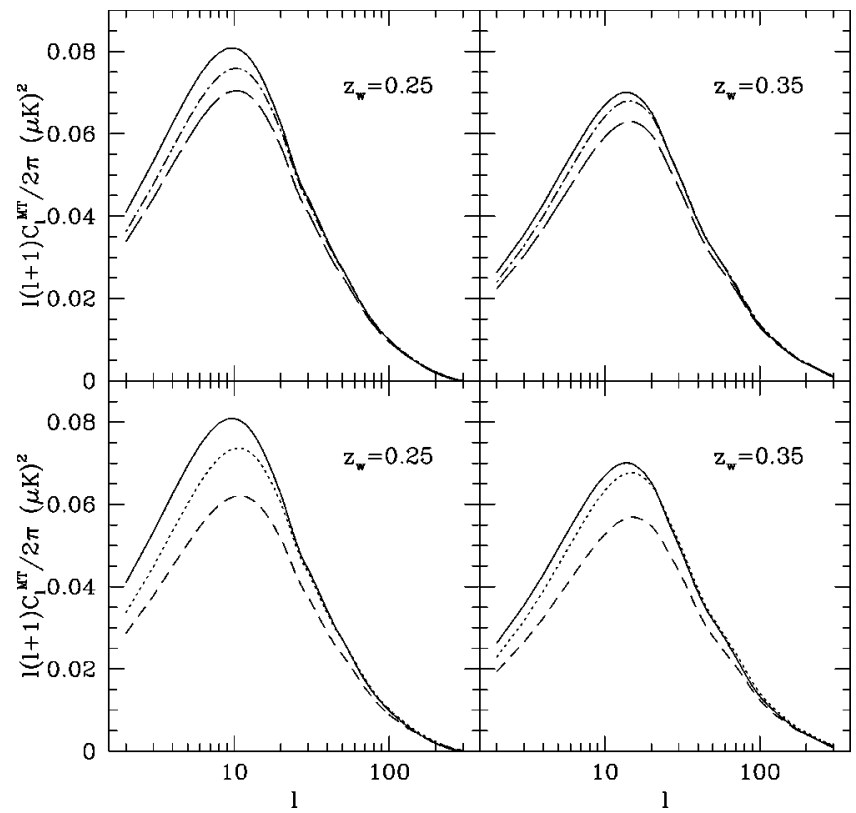

FIG. 10. Cross-correlation angular spectra for the same models as in Fig. 9.

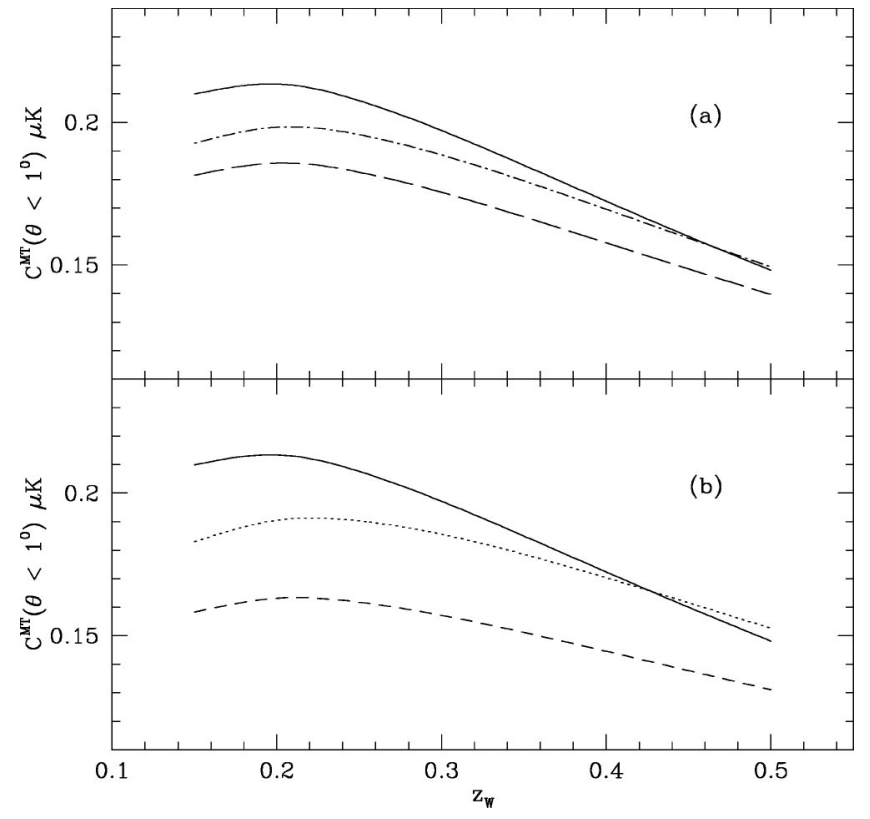

FIG. 11. $C^{M T}\left(0.1^{\circ}\right)$ vs $z_{w}$ for the models in Figs. 9 and 10: $\Lambda \mathrm{CDM}$ (solid), $\tilde{s}=2$ (dot-short dash), $w=-0.81$ (long dash), $\tilde{s}$ $=3$ (dot) and $w=-0.66$ (short dash).

$z_{w}$. (As is seen from Fig. 9, for angular separations less than about a degree, the plot is insensitive to the choice of $\theta$.) From Fig. 11 one can see that observations focusing on redshifts in the range $z_{w}=[0.2,0.4]$ have the best potential of detecting the time-dependence of $w$.

An interesting question is which scales give the dominant contribution to the cross-correlation. In Fig. 12 we plot the contribution per $\log (k)$ to the cross-correlation at $\theta=0.1^{\circ}$ for the $\Lambda \mathrm{CDM}, \tilde{s}=3$ and $w=-0.66$ models using $z_{w}=0.2$. Namely, we define a quantity $I(k)$ by

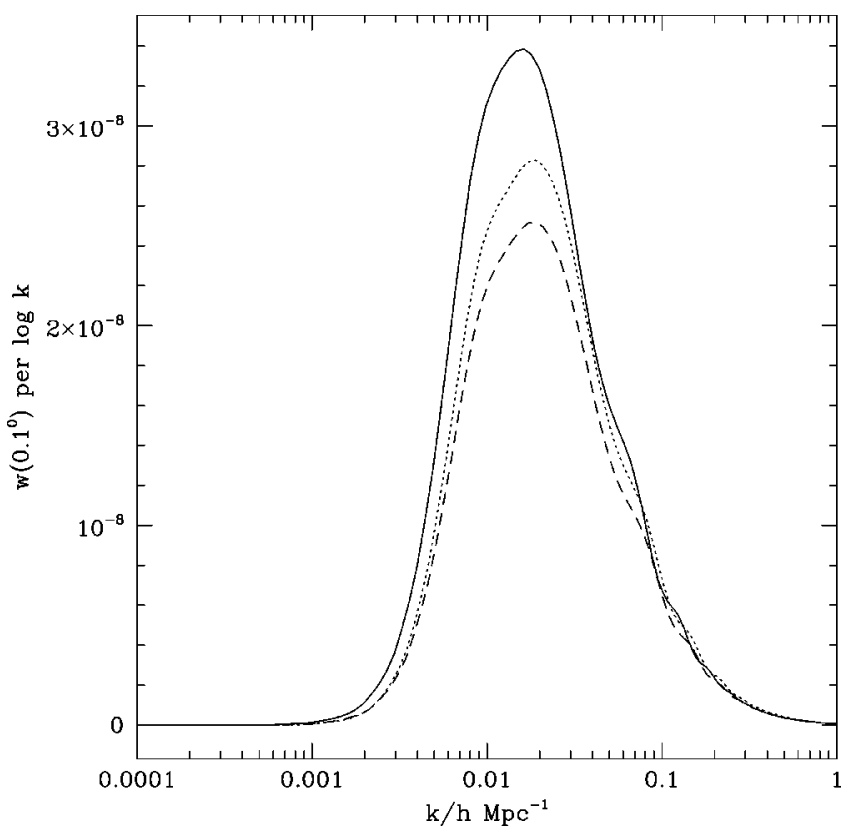

FIG. 12. $I(k)$ vs $\mathrm{k}$ for the $\Lambda \mathrm{CDM}$ (solid), $\tilde{s}=3$ (dot) and the $w=-0.66$ (short dash) models for $z_{w}=0.2$. 


$$
C^{M T}\left(0.1^{\circ}\right)=\int d(\ln k) I(k)
$$

The expression of $I(k)$ can be deduced from Eq. (A19) of the Appendix. As one can see from Fig. 12, $I(k)$ has a broad peak around $k \sim .01 \mathrm{~h} \mathrm{Mpc}^{-1}$, corresponding to length scales in the range $20-300 h^{-1} \mathrm{Mpc}$. This roughly coincides with the peak in the matter power spectrum, depicted in Fig. 8. For larger angular scales, it is still the same linear scales that dominate the integrand. However, for angles $\theta \gtrsim 10^{\circ}$ there is destructive interference between the modes and the correlation gradually disappears.

Let us now briefly consider the prospects for observation, and whether these will allow us to distinguish amongst different models. In practice, one correlates the CMB anisotropies with some data set (e.g. a galaxy survey) which is supposed to trace the underlying dark matter distribution. Of these two, the cleanest input is the CMB. Although very precise, current CMB data cannot separate the ISW contribution from the net anisotropy, which reduces the signal-tonoise ratio of the cross-correlation. There is hope, however, that such separation may be possible in the future, with measurements of CMB polarization towards galaxy clusters [37].

A prime source of uncertainty in the matter distribution is the bias factor. One way to proceed (which has been implicitly used e.g. in [5]) is the following. From the observed CMB autocorrelation, and following the steps we have described in the previous section, the matter power spectrum can be normalized. Comparing this to the autocorrelation function of any given matter survey, the bias factor can be inferred. Hence, from the cross-correlation of the survey with the CMB data one can infer the cross-correlation function of the matter distribution with the CMB. Note in particular that the predictions for the matter spectra for the $w=-0.66$ and $\tilde{s}=3$ models are practically indistinguishable (see Fig. 8). Hence, adjusting for the bias will preserve the relative difference between predictions for the cross-correlation for these two models. ${ }^{3}$

Error bars in current determinations of the crosscorrelation function [3-6] are still too large to distinguish between models. However, the results in [5] have been obtained by using just a small fraction of the sky, and the situation may improve considerably with fuller sky coverage. Also, the uncertainties in bias may be substantially reduced with further observations such as weak lensing surveys. The differences in the cross-correlation between the models considered in this section can be as large as $20 \%$, and they have a substantial dependence on redshift. Hence, it seems plau-

\footnotetext{
${ }^{3}$ It should be noted that at small angles (where the crosscorrelation with the matter power spectrum is maximal) and low redshifts, there is another contribution to the cross-correlation coming from the Sunjaev-Zeldovich (SZ) effect. Evidence for this contribution has already been reported in [5,7]. The SZ contribution depends on wavelength, and a number of future balloon experiments (as well as the Planck satellite) will be able to subtract this component out.
}

sible that they may fall within the range of detectability in a not too distant future.

\section{SUMMARY AND CONCLUSIONS}

Current efforts to understand the dark energy component of our universe often employ dynamical scalar fields. The dynamics of such scalar fields lead to variation of the dark energy equation of state parameter, $w$. We have considered astrophysical signatures of a varying equation of state, $w(z)$, in the context of doomsday models. There are two main issues that we have considered. First, can we tell if the average value of $w$ is different from -1 . Secondly, what astrophysical signatures are sensitive to the time variation of $w$ ?

To address both issues, we have considered autocorrelations of the CMB temperature anisotropies, the matter power spectrum, and cross-correlation of the anisotropy with matter fluctuations. The WMAP data are well reproduced by models with a different average value of $w$ by suitably adjusting the Hubble parameter, and therefore the CMB data cannot by themselves discriminate amongst different models. For a given $\mathrm{CMB}$, the present linear matter power spectrum is sensitive to the average value of $w(z)$, but not to its time dependence (see Fig. 8). Finally, the temperature-matter (TM) cross-correlation is sensitive to both the average value of $w$ and to the time variation (see Figs. 9, 10 and 11).

The TM cross-correlation requires accurate determination of both CMB anisotropies and the matter fluctuations. Ongoing CMB observations have been very successful at producing data with very small error bars and it is likely that the situation will improve even further in the coming years. Accurate surveys of the matter fluctuations are likely to be more challenging since there are unknowns such as the bias factor. However, since the CMB anisotropies and large-scale structure originate from the same density fluctuations, it is quite possible that a combination of the data can significantly reduce the uncertainties. We have shown that the optimal strategy for detecting a doomsday variation of $w(z)$ is to use a survey in the redshift range $[0.2,0.4]$.

Observations of supernovae over the next ten years are also likely to provide information on $w(z)$, as discussed in Ref. [22]. The advantage of planned supernovae observations are that they provide direct information on the Hubble expansion rate which is closely related to $w(z)$. Furthermore, the data will be of good quality, with small error bars. However, the observations may not be too sensitive to rapid variation of $w(z)$ at recent redshifts, the hallmark of the doomsday scenario. The TM cross-correlations, on the other hand, are sensitive to this time variation and may perhaps be used together with the supernovae observations to further constrain $w(z)$.

\section{ACKNOWLEDGMENTS}

We would like to thank Robert Caldwell, Michael Doran, Enrique Gaztanaga, Dragan Huterer, Andrew Jaffe, and Alex Vilenkin for very useful discussions and, additionally, M. Doran for cross-checking some of our preliminary results with his CMBEASY code [40]. The work of J.G. was sup- 
ported by CICYT Research Projects FPA2002-3598, FPA2002-00748, and DURSI 2001-SGR-0061. The work of T.V. was supported by DOE grant number DEFG0295ER40898 at CWRU.

\section{APPENDIX}

Here we explain details of our calculation of the crosscorrelation. Let us define

$$
\Delta(\hat{\mathbf{n}}) \equiv \frac{T(\hat{\mathbf{n}})-\bar{T}}{\bar{T}}
$$

and

$$
\delta(\hat{\mathbf{n}}) \equiv \frac{\rho(\hat{\mathbf{n}})-\bar{\rho}}{\bar{\rho}}
$$

where $T(\hat{\mathbf{n}})$ is the CMB temperature measured along the direction $\hat{\mathbf{n}}, \rho(\hat{\mathbf{n}})$ is the mass density along $\hat{\mathbf{n}},{ }^{4}$ and $\bar{T}$ and $\bar{\rho}$ are the averaged CMB temperature and the matter density. The temperature anisotropy due to the ISW effect is an integral over the conformal time:

$$
\Delta(\hat{\mathbf{n}})=\int_{\eta_{r}}^{\eta_{0}} d \eta e^{-\tau(\eta)}(\dot{\Phi}-\dot{\Psi})\left[\left(\eta_{0}-\eta\right) \hat{\mathbf{n}}, \eta\right],
$$

where $\eta_{r}$ is some initial time deep in the radiation era, $\eta_{0}$ is the time today, $\Phi$ and $\Psi$ are the Newtonian gauge gravitational potentials, ${ }^{5} \tau(\eta)$ is the opaqueness, which should, in principle, be included to account for the possibility of late reionization, and the dot denotes differentiation with respect to $\eta$.

The quantity $\delta(\hat{\mathbf{n}})$ contains contributions from astrophysical objects (e.g. galaxies) at different redshifts and can also be expressed as an integral over the conformal time:

$$
\delta(\hat{\mathbf{n}})=\int_{\eta_{r}}^{\eta_{0}} d \eta \frac{d z}{d \eta} W_{g}[z(\eta)] \delta\left(\left(\eta_{0}-\eta\right) \hat{\mathbf{n}}, \eta\right),
$$

where $W_{g}(z)$ is a normalized galaxy selection function.

We are interested in calculating the cross-correlation function

$$
C^{M T}(\theta) \equiv C^{M T}\left(\left|\hat{\mathbf{n}}_{1}-\hat{\mathbf{n}}_{2}\right|\right) \equiv\left\langle\Delta\left(\hat{\mathbf{n}}_{1}\right) \delta\left(\hat{\mathbf{n}}_{2}\right)\right\rangle,
$$

where the angular brackets denote ensemble averaging and $\theta$ is the angle between directions $\hat{\mathbf{n}}_{1}$ and $\hat{\mathbf{n}}_{2}$. Let us introduce

\footnotetext{
${ }^{4}$ In reality one divides the sky into pixels, with a direction $\hat{\mathbf{n}}$ assigned to each pixel, and counts the number of galaxies, $N(\hat{\mathbf{n}})$, inside each pixel. Then one can find the galaxy number overdensity inside each pixel: $[N(\hat{\mathbf{n}})-\bar{N}] / \bar{N}$, which would be related to $\rho(\hat{\mathbf{n}})$ up to a bias factor.

${ }^{5}$ Throughout this Appendix we work in the Newtonian gauge using conventions of, e.g., Ref. [38].
}

$$
r \equiv \eta_{0}-\eta
$$

The Fourier decomposition for $\dot{\Phi}(r \hat{\mathbf{n}}, \eta)$ can be written as

$$
\dot{\Phi}(r \hat{\mathbf{n}}, \eta)=\int \frac{d^{3} \mathbf{k}}{(2 \pi)^{3}} \dot{\Phi}(\mathbf{k}, \eta) e^{i \mathbf{k} \cdot \hat{\mathbf{n}} r}
$$

and similarly for $\Psi(r \hat{\mathbf{n}}, \eta)$ and $\delta_{g}(r \hat{\mathbf{n}}, \eta)$. We can write

$$
\begin{aligned}
C^{M T}(\theta)= & \int_{\eta_{r}}^{\eta_{0}} d \eta_{1} \int_{\eta_{r}}^{\eta_{0}} d \eta_{2} \dot{z}\left(\eta_{2}\right) e^{-\tau\left(\eta_{1}\right)} W_{g}\left[z\left(\eta_{2}\right)\right] \\
& \times \int \frac{d^{3} \mathbf{k}}{(2 \pi)^{3}} \int \frac{d^{3} \mathbf{k}^{\prime}}{(2 \pi)^{3}} e^{i \mathbf{k} \cdot \hat{\mathbf{n}}_{1} r_{1}} e^{i \mathbf{k}^{\prime} \cdot \hat{\mathbf{n}}_{2} r_{2}} \\
& \times\left\langle\left[\Phi\left(\mathbf{k}, \eta_{1}\right)-\Psi\left(\mathbf{k}, \eta_{1}\right)\right] \delta\left(\mathbf{k}^{\prime}, \eta_{2}\right)\right\rangle .
\end{aligned}
$$

Since the time-evolution of each Fourier mode only depends on the magnitude $k=|\mathbf{k}|$, we can separate the directional and time dependence as

$$
\begin{gathered}
\Phi(\mathbf{k}, \eta) \equiv \Phi\left(\mathbf{k}, \eta_{r}\right) \phi(k, \eta) \\
\Psi(\mathbf{k}, \eta) \equiv \Psi\left(\mathbf{k}, \eta_{r}\right) \psi(k, \eta) \\
\delta(\mathbf{k}, \eta) \equiv \delta\left(\mathbf{k}, \eta_{r}\right) \widetilde{\delta}(k, \eta)
\end{gathered}
$$

Hence, we can write $\Phi$ and $\dot{\Psi}$ as

$$
\begin{aligned}
& \dot{\Phi}(\mathbf{k}, \eta) \equiv \Phi\left(\mathbf{k}, \eta_{r}\right) \dot{\phi}(k, \eta) \\
& \dot{\Psi}(\mathbf{k}, \eta) \equiv \Psi\left(\mathbf{k}, \eta_{r}\right) \dot{\psi}(k, \eta) .
\end{aligned}
$$

Consequently, the quantity $\left\langle\left[\Phi\left(\mathbf{k}, \eta_{1}\right)-\Psi\left(\mathbf{k}, \eta_{1}\right)\right]\right.$ $\left.\delta\left(\mathbf{k}^{\prime}, \eta_{2}\right)\right\rangle$ can be separated into the initial power spectra, which contain all the information relevant to the ensemble averaging, and the time-evolving part which is the same for all members of the ensemble:

$$
\begin{aligned}
\left\langle\left[\dot{\Phi}\left(\mathbf{k}, \eta_{1}\right)-\dot{\Psi}\left(\mathbf{k}, \eta_{1}\right)\right] \delta\left(\mathbf{k}^{\prime}, \eta_{2}\right)\right\rangle \\
=\left\langle\Phi\left(\mathbf{k}, \eta_{r}\right) \delta\left(\mathbf{k}^{\prime}, \eta_{r}\right)\right\rangle \dot{\phi}\left(k, \eta_{1}\right) \widetilde{\delta}\left(k^{\prime}, \eta_{2}\right) \\
\quad-\left\langle\Psi\left(\mathbf{k}, \eta_{r}\right) \delta\left(\mathbf{k}^{\prime}, \eta_{r}\right)\right\rangle \dot{\psi}\left(k, \eta_{1}\right) \widetilde{\delta}\left(k^{\prime}, \eta_{2}\right) .
\end{aligned}
$$

We take $\eta_{r}$ to be a sufficiently early time in the radiation era when all modes under consideration were superhorizon. Then, for adiabatic initial conditions, the growing mode solutions for $\delta, \Phi$ and $\Psi$ are related to each other via [39]

$$
c_{\delta \Psi} \equiv \frac{\delta}{\Psi}=-\frac{3}{2}, \quad c_{\Phi \Psi} \equiv \frac{\Phi}{\Psi}=-\left(1+\frac{2}{5} R_{\nu}\right),
$$

where $R_{\nu} \equiv \rho_{\nu} /\left(\rho_{\gamma}+\rho_{\nu}\right)$ and $\rho_{\nu}$ is the energy density in relativistic neutrinos. For $N_{\nu}$ flavors of relativistic neutrinos (we take $N_{\nu}=3$ ), after electron-positron pair annihilation, $\rho_{\nu} / \rho_{\gamma}=\left(7 N_{\nu} / 8\right)(4 / 11)^{4 / 3}$. This allows us to write 


$$
\begin{aligned}
& \left\langle\left[\dot{\Phi}\left(\mathbf{k}, \eta_{1}\right)-\dot{\Psi}\left(\mathbf{k}, \eta_{1}\right)\right] \delta\left(\mathbf{k}^{\prime}, \eta_{2}\right)\right\rangle \\
& =c_{\delta \Psi}\left\langle\Psi\left(\mathbf{k}, \eta_{r}\right) \Psi\left(\mathbf{k}^{\prime}, \eta_{r}\right)\right\rangle \\
& \quad \times\left[c_{\Phi \Psi} \dot{\phi}\left(k, \eta_{1}\right)-\dot{\psi}\left(k, \eta_{1}\right)\right] \widetilde{\delta}\left(k^{\prime}, \eta_{2}\right) .
\end{aligned}
$$

From homogeneity of space it follows that

$$
\left\langle\Psi\left(\mathbf{k}, \eta_{r}\right) \Psi\left(\mathbf{k}^{\prime}, \eta_{r}\right)\right\rangle=(2 \pi)^{3} \delta^{(3)}\left(\mathbf{k}+\mathbf{k}^{\prime}\right) P_{\Psi}(k),
$$

where $P_{\Psi}(k)$ is the primordial gravitational power spectrum related to the more frequently used curvature power spectrum $P_{\mathcal{R}} \equiv 2 \pi^{2} \Delta_{\mathcal{R}}^{2} / k^{3}$ via $[32,38]$

$$
P_{\Psi}(k)=\frac{9}{25} P_{\mathcal{R}}(k)=\frac{9}{25} \frac{2 \pi^{2}}{k^{3}} \Delta_{\mathcal{R}}^{2} .
$$

To the best of our knowledge, in all previous literature that contained calculations of the cross-correlation, it was the matter power spectrum at recent redshifts that was used, rather than the primordial spectrum. One can do that if fluctuations in the dark energy $\delta \rho_{D}$ are much smaller than those in cold dark matter, $\delta \rho_{c d m}$ : for $z \rightarrow 0$, one has

$$
\Phi-\Psi \approx-\frac{H^{2}}{k^{2}}\left(\delta \rho_{c d m}+\delta \rho_{D}\right)
$$

and usually one proceeds by assuming that $\delta \rho_{D} \ll \delta \rho_{c d m}$ in the equation above. While this is not necessarily an invalid condition, working with the primordial spectrum allows us to use exact relations (A12), valid deep in the radiation era, when all relevant modes are outside the horizon and dark energy fluctuations are negligible, and avoid the need for additional assumptions. It turns out, however, that, while on scales $k \leqq 0.001 \mathrm{~h} \mathrm{Mpc}^{-1}$ and larger the fluctuations in the dark energy can be as large as $10 \%$, on scales $k$ $\sim .01 \mathrm{~h} \mathrm{Mpc}^{-1}$, where the cross-correlation is important, the contribution of dark energy perturbations is rather small, less than $1 \%$.

Using Eqs. (A13) and (A14) we can now rewrite Eq. (A8) as

$$
\begin{aligned}
C^{M T}(\theta)= & \frac{9}{25} \int_{\eta_{r}}^{\eta_{0}} d \eta_{1} \int_{\eta_{r}}^{\eta_{0}} d \eta_{2} \dot{z}\left(\eta_{2}\right) e^{-\tau\left(\eta_{1}\right)} W_{g}\left[z\left(\eta_{2}\right)\right] \\
& \times \int \frac{d^{3} \mathbf{k}}{4 \pi k^{3}} \Delta_{\mathcal{R}}^{2}(k) e^{i \mathbf{k} \cdot\left(\hat{\mathbf{n}}_{1} r_{1}-\hat{\mathbf{n}}_{2} r_{2}\right)} F\left(k, \eta_{1}, \eta_{2}\right),
\end{aligned}
$$

where we have defined

$$
F\left(k, \eta_{1}, \eta_{2}\right) \equiv c_{\delta \Psi}\left[c_{\Phi \Psi} \dot{\phi}\left(k, \eta_{1}\right)-\dot{\psi}\left(k, \eta_{1}\right)\right] \widetilde{\delta}\left(k, \eta_{2}\right)
$$

Decomposing the exponents in Eq. (A17) into spherical functions and some manipulations lead to

$$
\begin{aligned}
C^{M T}(\theta)= & \frac{9}{25} \int_{\eta_{r}}^{\eta_{0}} d \eta_{1} \int_{\eta_{r}}^{\eta_{0}} d \eta_{2} \dot{z}\left(\eta_{2}\right) e^{-\tau\left(\eta_{1}\right)} W_{g}\left[z\left(\eta_{2}\right)\right] \\
& \times \int \frac{d k}{k} \Delta_{\mathcal{R}}^{2}(k) \frac{\sin (k R)}{k R} F\left(k, \eta_{1}, \eta_{2}\right),
\end{aligned}
$$

where $R \equiv \sqrt{r_{1}^{2}+r_{2}^{2}-2 r_{1} r_{2} \cos \theta}$. In addition, if one were to use the expression (A19), one would have to subtract the monopole and dipole contributions to $C^{M T}(\theta)$. This can be achieved by taking

$$
\begin{aligned}
\frac{\sin (k R)}{k R} \rightarrow & \frac{\sin (k R)}{k R}-\frac{\sin k r_{1}}{k r_{1}} \frac{\sin k r_{2}}{k r_{2}} \\
& -\frac{3}{k^{2} r_{1} r_{2}}\left(\frac{\sin k r_{1}}{k r_{1}}-\cos k r_{1}\right)\left(\frac{\sin k r_{2}}{k r_{2}}-\cos k r_{2}\right) .
\end{aligned}
$$

In practice, one wants to avoid evaluating double time integrals in Eq. (A19). A common way to reduce them to a single time integration is to use the so-called small angle $(\theta \ll 1)$ and small separation $\left(\left|r_{1}-r_{2}\right| \ll r_{1}\right)$ approximations [29]. These approximations were used in e.g. Refs. [5,6]. One can change the integration variables to $x=r_{1}-r_{2}$ and $r=\left(r_{1}+r_{2}\right) / 2$ [or, equivalently, to $\left.\eta=\left(\eta_{1}+\eta_{2}\right) / 2\right]$ and write, in this approximation,

$$
\begin{aligned}
C^{M T}(\theta) \approx & \frac{9}{25} \int_{\eta_{r}}^{\eta_{0}} d \eta \dot{z} e^{-\tau(\eta)} W_{g}[z(\eta)] \\
& \times \int \frac{d k}{k} \Delta_{\mathcal{R}}^{2}(k) F(k, \eta, \eta) \int_{-2 r}^{2 r} d x \frac{\sin (k R)}{k R},
\end{aligned}
$$

where $R \approx \sqrt{x^{2}+r^{2} \theta^{2}}$. The integral over $x$ could be evaluated analytically if one were allowed to replace the $[-2 r, 2 r]$ limits by $[-\infty, \infty]$. One can assume that $2 r$ is sufficiently large $(r>1 / k \theta)$ on relevant scales for that replacement to be appropriate and use

$$
\int_{-\infty}^{\infty} d x \frac{\sin (k R)}{k R}=\frac{\pi}{k} J_{0}(k r \theta)
$$

to obtain the following form:

$$
\begin{aligned}
C^{M T}(\theta) \approx & \frac{9}{25} \int_{\eta_{r}}^{\eta_{0}} d \eta \dot{z} e^{-\tau(\eta)} W_{g}(z(\eta)) \\
& \times \int \frac{\pi d k}{k^{2}} \Delta_{\mathcal{R}}^{2}(k) J_{0}\left(k \theta\left[\eta_{0}-\eta\right]\right) F(k, \eta, \eta) .
\end{aligned}
$$

While the approximate expression (A23) can be useful for analytical estimates, it gives an error of order $2-4 \%$ on the scales of interest and we shall not resort to it.

Instead of evaluating the expression (A19) directly, from a computational point of view, it is advantageous to decompose it into Legendre series, compute the individual coeffi- 
cients of the decomposition, and then sum the series. Namely, Eq. (A19) can be written as

$$
C^{M T}(\theta)=\sum_{l=2}^{\infty} \frac{2 \ell+1}{4 \pi} C^{M T}{ }_{\ell} P_{\ell}(\cos \theta),
$$

where we do not include the monopole and dipole terms in the sum, and where $C^{M T}{ }_{\ell}$ can be written as

$$
C^{M T}{ }_{\ell}=4 \pi \frac{9}{25} \int \frac{d k}{k} \Delta_{\mathcal{R}}^{2} T^{I S W}(k) M_{\ell}(k),
$$

with functions $T^{I S W}(k)$ and $M_{\ell}(k)$ defined as

$$
\begin{gathered}
T_{\ell}^{I S W}=\int_{\eta_{r}}^{\eta_{0}} e^{-\tau(\eta)} d \eta j_{\ell}\left(k\left[\eta-\eta_{0}\right]\right)\left(c_{\Phi \Psi} \dot{\phi}-\dot{\psi}\right) \\
M_{\ell}=c_{\delta \Psi} \int_{\eta_{r}}^{\eta_{0}} d \eta j_{\ell}\left(k\left[\eta-\eta_{0}\right]\right) \dot{z} W_{g}[z(\eta)] \widetilde{\delta}(k, \eta),
\end{gathered}
$$

where $j_{\ell}(\cdot)$ are spherical Bessel functions. One can use CMBFAST [28], with minor modifications, to compute functions $T^{I S W}$ and $M_{\ell}$ and to normalize $\Delta_{\mathcal{R}}^{2}(k)$.
[1] A. Riess et al., Astron. J. 116, 1009 (1998).

[2] S. Perlmutter et al., Astrophys. J. 517, 565 (1999).

[3] S.P. Boughn, R.G. Crittenden, and G.P. Koehrsen, Astrophys. J. 580, 672 (2002); S.P. Boughn and R.G. Crittenden, Phys. Rev. Lett. 88, 021302 (2001); astro-ph/0305001.

[4] M.R. Nolta et al., astro-ph/0305097.

[5] P. Fosalba and E. Gaztanaga, astro-ph/0305468; P. Fosalba, E. Gaztanaga, and F. Castander, Astrophys. J. Lett. 597, L89 (2003).

[6] R. Scranton et al., astro-ph/0307335.

[7] N. Afshordi, Y.S. Loh, and M.A. Strauss, astro-ph/0308260.

[8] A.D. Linde, Rep. Prog. Phys. 47, 925 (1984).

[9] A. Vilenkin, Phys. Rev. Lett. 74, 846 (1985).

[10] G. Efstathiou, Mon. Not. R. Astron. Soc. 274, L73 (1995).

[11] S. Weinberg, Phys. Rev. Lett. 59, 2607 (1987).

[12] H. Martel, P.R. Shapiro, and S. Weinberg, Astrophys. J. 492, 29 (1998).

[13] J. Garriga, M. Livio, and A. Vilenkin, Phys. Rev. D 61, 023503 (2000).

[14] S.A. Bludman, Nucl. Phys. A663, 865 (2000).

[15] J. Garriga and A. Vilenkin, Phys. Rev. D 61, 083502 (2000).

[16] A.D. Linde, in 300 Years of Gravitation, edited by S.W Hawking and W. Israel (Cambridge University Press, Cambridge, 1987).

[17] J. Garriga and A. Vilenkin, Phys. Rev. D 67, 043503 (2003).

[18] S. Dimopoulos and S. Thomas, hep-th/0307004.

[19] This possibility was pointed out in [17]. However, an error in the analysis of the prior distribution after inflation led to the conclusion that it always favors small values of $s$, implying that the equation of state $w=-1$ should be expected in general. This has been corrected in Ref. [21] where it is shown that both situations are possible, depending on the shape of the multifield potential $V\left(\phi_{a}\right)$. In particular, there is a wide class of models with prior distributions favoring large $s$.

[20] R. Kallosh, A. Linde, S. Prokushkin, and M. Shmakova, Phys. Rev. D 66, 123503 (2002).
[21] J. Garriga, A.D. Linde, and A. Vilenkin, hep-th/0310034.

[22] R. Kallosh, J. Kratochvil, A. Linde, E.V. Linder, and M. Shmakova, J. Cosmol. Astropart. Phys. 10, 015 (2003).

[23] R.R. Caldwell, R. Dave, and P.J. Steinhardt, Phys. Rev. Lett. 80, 1582 (1998).

[24] R.G. Crittenden and N. Turok, Phys. Rev. Lett. 76, 575 (1996).

[25] H.V. Peiris and D.N. Spergel, Astrophys. J. 540, 605 (2000).

[26] A. Cooray, Phys. Rev. D 65, 103510 (2002).

[27] R. Bean and O. Dore, Phys. Rev. D (to be published), astro-ph/0307100.

[28] M. Zaldariaga and U. Seljak, Astrophys. J. 469, 437 (1996); http://www.cmbfast.org

[29] D. Limber, Astrophys. J. 119, 655 (1954).

[30] J.R. Bond and G. Efstathiou, Mon. Not. R. Astron. Soc. 304, 75 (1999)

[31] WMAP Team, D. Spergel et al., Astrophys. J., Suppl. 148, 175 (2003).

[32] WMAP Team, L. Verde et al., Astrophys. J., Suppl. 148, 195 (2003); WMAP Team, H.V. Peiris et al., ibid. 148, 213 (2003).

[33] The Supernova Cosmology Project, R.A. Knop et al., astro-ph/0309368.

[34] E.V. Linder and D. Huterer, Phys. Rev. D 67, 081303 (2003); D. Huterer and M.S. Turner, ibid. 64, 123527 (2001); R.R. Caldwell and M. Doran, astro-ph/0305334.

[35] HST Key Project, W.L. Freedman et al., Astrophys. J. 553, 47 (2001).

[36] A. Liddle and D.H. Lyth, Cosmological Inflation and Largescale Structure (Cambridge University Press, New York, 2000).

[37] A. Cooray, D. Huterer, and D. Baumann, Phys. Rev. D (to be published), astro-ph/0304268.

[38] H. Kodama and M. Sasaki, Prog. Theor. Phys. Suppl. 78, 1 (1984).

[39] C.-P. Ma and E. Bertschinger, Astrophys. J. 455, 7 (1995).

[40] M. Doran, astro-ph/0302138; http://www.cmbeasy.org 